\title{
Sputtering of water ice films: a re-assessment with singly and doubly charged oxygen and argon ions, molecular oxygen, and electrons
}

\author{
A. Gallia,1, A. Vorburger ${ }^{\mathrm{a}}$, P. Wurz ${ }^{\mathrm{a}}$, M. Tulej ${ }^{\mathrm{a}}$ \\ ${ }^{a}$ Physikalisches Institut, University of Bern, Bern, Switzerland
}

\begin{abstract}
We studied the erosion rates from thin water ice films on a microbalance upon irradiation with ions $\left(\mathrm{O}^{+}, \mathrm{O}_{2}^{+}, \mathrm{O}^{2+}, \mathrm{Ar}^{+}\right.$, and $\left.\mathrm{Ar}^{2+}\right)$ and electrons at energies between $0.1 \mathrm{keV}$ and $80 \mathrm{keV}$. The results with $\mathrm{O}^{+}$and $\mathrm{Ar}^{+}$irradiation confirm previous results of other research groups that relied on the same experiment set-up. In addition, we assessed how the ice film thickness affects the results and we compared the results for singly versus doubly charged ions and for $\mathrm{O}^{+}$versus $\mathrm{O}_{2}^{+}$ions. The irradiation with $1 \mathrm{keV}$ and $3 \mathrm{keV}$ electrons offer the first experimental results at these energies. Our results confirm theoretical predictions that the yield per impacting electron does not increase with energy ad infinitum but rather levels off between 0.1 and 1 $\mathrm{keV}$. The results for ion and electron sputtering have important implications for atmosphere-less icy bodies in a plasma environment. We briefly discuss the implications for the icy moons of Jupiter. Finally, the experiments also allow us to assess the viability of two methods to measure the erosion rate in the case that the icy sample cannot be attached on a microbalance. This is an important step for future laboratory studies where regolith ice samples and their reaction to particle irradiation are to be characterized.
\end{abstract}

Keywords: Ices, Jupiter satellites, Experimental techniques

\section{Introduction}

When water ice is irradiated with energetic ions or electrons, the energy of the impactor may eject particles. This so-called sputtering may be straight-

\footnotetext{
${ }^{1}$ Corresponding author, E-mail address: andre.galli@space.unibe.ch
} 
forward, i.e., an ion knocks off one or several water molecules, or it may be a 5 two-stage process with the irradiation first causing chemical reactions inside the ice (so-called radiolysis, Johnson et al. (2004); Cassidy et al. (2010)) and subsequently releasing the radiolysis products from the surface. The sputtering yield denotes in both cases the number of water molecules or equivalents (if $\mathrm{H}_{2} \mathrm{O}$ reacted to $\mathrm{H}_{2}$ and $\mathrm{O}_{2}$, for instance) per impacting ion or electron. Knowing this yield and the chemical and energetic composition of the ejecta over a wide range of parameters is important to understand any ice-covered celestial body. The astrophysical applications we are most interested in are the icy moons of Jupiter. For these bodies, the sputtering yields and the plasma environment determine the density and composition of their atmospheres (see Johnson et al. (2004) for a review).

Sputtering yields can be determined theoretically or with laboratory experiments. The most common experimental method used so far consists of vapour-depositing a water ice film on a microbalance and then irradiating the ice film with an ion beam (see Famá et al. (2008); Johnson et al. (2009); Cassidy et al. 2013 for meta-studies and compilations of such experiments). The microbalance measures accurately the mass being accreted or lost. However, it remained unclear whether such thin water ice films are a good proxy for the deep, porous regolith of real surfaces. The potential effect of porosity, for instance, introduces an uncertainty of $70 \%$ (U. Raut, personal communication 2016, and comparing Cassidy and Johnson (2005) with Cassidy et al. (2013)). Depending on the specific moon to be studied, also salts, nonwater ices, silicates, and a frost layer may be present (Calvin et al., 1995; Domingue and Verbiscer, 1997; Grundy et al., 1999; Johnson et al., 2004; Shi et al., 2010). In Galli et al. (2016) we presented experiments conducted 30 with a $0.9 \mathrm{~cm}$ deep sample of icy regolith with a density of $0.3 \mathrm{~g} \mathrm{~cm}^{-3}$. That approach allows us to conduct sputtering experiments with a thick porous sample. However, the sputtering yield from such an ice sample that cannot be attached to a microbalance must be measured with another method. The emitted particles are difficult to detect since most of them are neutral and have energies too low for energetic neutral particle detectors (Wurz, 2000). Either the residual gas pressure in the vacuum chamber must be monitored to reveal a pressure rise (method used by Vidal et al. (2005); Galli et al. (2016)) or a cooled microbalance or any similar device must be mounted opposite the irradiated ice surface to collect a part of the emitted particles.

40 For this study, we sputtered thin water ice films from a microbalance. After a recapitulation of the theory of ice sputtering (Section 2), we describe 
the experiment set-up in Section 3. We then present the sputtering results for various ion species (Section 4.1). Our experiments reproduce available data from other research groups (for $\mathrm{O}^{+}, \mathrm{O}^{2+}$, and $\mathrm{Ar}^{+}$ions) and provide new results for ion species $\left(\mathrm{O}_{2}^{+}\right.$, and $\left.\mathrm{Ar}^{2+}\right)$ that have, to our knowledge, not been tested before. In Section 4.2, we show the ion sputtering results for the double microbalance set-up, followed by the electron sputtering results in Section 4.3. As electron energies, we chose $0.1 \mathrm{keV}$ in analogy to Orlando and Sieger (2003) and then proceeded to energies hitherto not studied in experiments $(1 \mathrm{keV}$ and $3 \mathrm{keV})$. Since we accompanied all these measurements with gas pressure measurements or with a secondary microbalance above the first one, we also can compare the accuracy of these two measurement methods (Section 4.4). The paper is concluded with a summary of results and implications for the icy moons of Jupiter and for future laboratory work (Section 5).

\section{Theory}

To relate our sputtering yields to previous experimental studies we will rely on the semi-empirical formula derived by Famá et al. (2008). It serves as a summary of previous sputtering experiments with dense water ice films. For ion energies below $10 \mathrm{keV}$, the sputtering yield of ions in water ice can be described by a cascade of elastic collisions, whereas at higher energies, the socalled electronic sputtering dominates. The total sputtering yield (number of water molecules per incident ion) is the sum of the two contributions. Famá et al. (2008) derived an expression including both contributions, which fit their laboratory measurements and results of other research groups (Johnson and $\mathrm{Liu}, 2010$ for $\mathrm{H}^{+}, \mathrm{He}^{+}, \mathrm{N}^{+}, \mathrm{O}^{+}, \mathrm{Ne}^{+}$, and $\mathrm{Ar}^{+}$beams:

$Y\left(E, m_{1}, Z_{1}, \theta, T\right)=\frac{1}{U_{i}}\left(\frac{3}{4 \pi^{2} C_{0}} \alpha S_{n}+\eta S_{e}^{2}\right)\left(1+q_{i} \exp \left(-\frac{E_{a}}{k_{B} T}\right)\right) \cos ^{-f}(\theta)$

Equation 1 quantifies the sputtering yield as a sum of elastic and electronic sputtering, described by the nuclear stopping power $S_{n}\left(E, m_{1}, Z_{1}\right)$ and the electronic stopping power $S_{e}\left(E, m_{1}, Z_{1}\right)$. The sputtering yield depends on energy $E$, mass of impactor $m_{1}$, atomic number of impactor $Z_{1}$, the incidence angle $\theta$ relative to the surface normal, and temperature $T$. The temperaturedependent term with the activation energy $E_{a}$ (Reimann et al., 1984) becomes dominant above $T=120 \mathrm{~K}$ and is due to radiolysis and subsequent release 
of $\mathrm{H}_{2}$ and $\mathrm{O}_{2}$ (Johnson et al., 2004; Famá et al., 2008; Teolis et al., 2009). 75 At lower temperatures, the ejecta are predominantly $\mathrm{H}_{2} \mathrm{O}$ molecules. The ratio of released $\mathrm{O}_{2}$ to $\mathrm{H}_{2} \mathrm{O}$ for $\mathrm{O}^{+}$ions irradiating $100 \mathrm{~K}$ water ice varies between 0.25 and 0.05 for the energy range of 1 to $100 \mathrm{keV}$ (Teolis et al., 2016). For $U_{i}$, the sublimation energy of water $(0.45 \mathrm{eV})$ is assumed. The effective cross-section for low energy recoils, $C_{0}=1.3 \AA^{2}$, the activation energy, $E_{a}=0.06 \pm 0.01 \mathrm{eV}$, and $q_{i}=220$ are constants. The parameter describing the angular dependence calculates to $f=1.78$ for $\mathrm{Ar}^{+}$. From the angular dependence in Eq. 1 one expects an order of magnitude higher sputtering yields at ion incidence angles around $80^{\circ}$ than for perpendicular ion impacts. The condition is that the ice sample is microscopically smooth. Küstner et al. (1998) studied graphite surfaces of varying roughness on a $\mu \mathrm{m}$ scale and found that the sputtering yield increased only by a factor of 2.5 when the ion incidence angle increased from $0^{\circ}$ to $80^{\circ}$. For a smooth graphite surface, they confirmed that $Y$ increases by more than a decade. In the following section, we will compare our new experiment results for $\mathrm{Ar}^{+}$to the predictions in Eq. 1.

For electron irradiation we expect from previous experiments (see Johnson et al. (2013); Teolis et al. (2009) for reviews) that most of the lost mass will not be emitted as water but as $\mathrm{H}_{2}$ and $\mathrm{O}_{2}$ instead, with the yield of $\mathrm{H}_{2}$ roughly two times the $\mathrm{O}_{2}$ yield. Teolis et al. (2016) predicted, based on experiments (Baragiola et al., 2002; Boring et al., 1983; Orlando and Sieger, 2003) that the $\mathrm{O}_{2}$ sputtering yield should linearly increase until $100 \mathrm{eV}$ but then should turn over around $400 \mathrm{eV}$, admitting that "measurements above $100 \mathrm{eV}$ are lacking". Teolis et al. (2016) calculated the following sputtering yield for $\mathrm{O}_{2}$ equivalents per impacting electron:

$$
Y_{O 2}(E, T, \theta)=\frac{E}{U_{O 2}} \frac{x_{0}}{d \cos \theta}\left[1-\exp \left(-\frac{d \cos \theta}{x_{0}}\right)\right]\left(1+q_{O 2} \exp \left(\frac{-E_{a}}{k_{B} T}\right)\right)
$$

with $d \cos \theta$ the penetration depth, $x_{0}=2.8 \mathrm{~nm}$ the thickness of the surface layer where $\mathrm{O}_{2}$ escape is efficient, $U_{O 2}=200 \mathrm{eV}$ at low temperatures to 80 $\mathrm{K}$, and $q_{O 2}=1000 \pm 100$ the fit variable for the thermal dependence. The sputtering yield in Equation 2 decreases for energies above $400 \mathrm{eV}$ because the penetration depth becomes much larger than the surface layer thickness $x_{0}$ at this energy. The penetration depth $d$ of electrons in water ice can be approximated (Johnson, 1990; Hand and Carlson, 2011) by 


$$
d \approx R_{0} E^{\alpha}
$$

with $E$ electron energy in units of $\mathrm{keV}, R_{0}=46 \mathrm{~nm}$ and $\alpha=1.76$.

\section{Experiment set-up}

The MEFISTO test facility for space instrument calibration consists of a

110 2001). We also used this facility for the sputtering experiments with a deep porous ice sample described in (Galli et al. 2016). We did not insert thick ice samples into the chamber for the present study. Instead, we deposited de-ionized water from an omnidirectional vapour source on the cooled surface Research). The surface of the microbalance was $45^{\circ}$ tilted with respect to the normal. Under these conditions and temperatures around $93 \mathrm{~K}$, most of the deposited ice will remain amorphous throughout the experiments and the porosity will amount to roughly 0.2 before the ice is irradiated (Mitchell varying intensity and energy. The current and the dimension of the beams were monitored with a Faraday cup $6 \mathrm{~cm}$ away from the microbalance. The Faraday cup was operated at a positive potential of $18 \mathrm{~V}$ (Galli et al., 2016), which is negligible compared to the energy of the beams.

its frequency and the deposited mass. To convert measured frequency rates into physical units, the following numbers apply: the microbalance sensitivity is $S=1.61 \times 10^{9} \mathrm{~Hz} \mathrm{~g}^{-1}$, the area of the active surface is $0.316 \mathrm{~cm}^{2}$, and a monolayer of $\mathrm{H}_{2} \mathrm{O}$ atoms is $3 \times 10^{-10} \mathrm{~m}$ in thickness. For the average $\mathrm{cm}^{-3}$ (Famá et al. 2008) and thus a porosity $<0.05$. This implies that a 14 $\mathrm{Hz}$ frequency difference corresponds to the loss of one monolayer of ice. The residual water pressure during irradiation experiments was a few $10^{-9}$ mbar, corresponding to a deposition rate of roughly $40 \mathrm{~Hz} \mathrm{~h}^{-1}$. We subtracted this which the change in frequency rate due to irradiation was much larger than the difference of frequency rates before and after irradiation.

We positioned the microbalances in two different ways on a plate cooled with liquid nitrogen: For most experiments we placed one microbalance at 
the very front of the cooling plate and irradiated its active surface. We then analysed the frequency of the irradiated microbalance and the pressure in the vacuum chamber. With the single microbalance set-up we wanted to verify previous sputtering experiments for $\mathrm{Ar}^{+}$and $\mathrm{O}^{+}$and check sputtering yields for other ion species that had not been studied before. We also irradiated the ice films with electrons to obtain sputtering yields and composition for electrons irradiating water ice. The last goal of this set-up was to assess how accurately the pressure rise measured in the vacuum chamber correlated with the erosion rate derived from the microbalance. The residual pressure was measured with a Stabil-Ion pressure gauge (manufacturer: GranvillePhillips) at intervals of one second. The gauge was placed $1 \mathrm{~m}$ away from the microbalance with no direct line of sight between the two. The electron irradiation experiments were also analysed with a mass spectrometer (HAL quadrupole gas analyser, manufacturer: Hiden Analytical) mounted $70 \mathrm{~cm}$ above the microbalances.

Three additional days of experiments were dedicated to a double microbalance set-up: the second balance was mounted on the same cooling plate, facing the primary microbalance at a distance of $2.5 \mathrm{~cm}$ between the active surfaces. This way, the secondary microbalance caught water molecules ejected from the primary microbalance when it was irradiated with ions. The double set-up allowed us to constrain the opening angle of the sputtering cone and the sticking probability of the ejected $\mathrm{H}_{2} \mathrm{O}$ molecules. It also served as a test if one could determine the primary sputtering yield with this detection method without the information from the primary microbalance. Contrary to $\mathrm{H}_{2} \mathrm{O}, \mathrm{O}_{2}$ and $\mathrm{H}_{2}$ would not permanently stick to the microbalance at the temperatures around $90 \mathrm{~K}$, but the latter molecules contribute little to the total mass of ejecta at these temperatures (Equation 1 .

\section{Results}

\subsection{Ion sputtering yields for single microbalance set-up}

The yields measured with this setup represent the loss rate of water ice on the microbalance and include all ejected species $\left(\mathrm{H}_{2} \mathrm{O}\right.$ plus smaller fractions of $\mathrm{O}_{2}$ and $\mathrm{H}_{2}$ ). We deposited an ice film, irradiated it about ten times with ion beams of different energy and flux, then added a fresh layer of ice. Since the deposition rate was orders of magnitudes higher than the erosion rate during irradiation, this process allowed us to distinguish between potential effects of irradiation dose and ice film thickness. These effects are weak for 
our parameter range as will be explained in the two following paragraphs. From then onwards we no longer discriminated against ion beam intensity or ice film thickness; we grouped the 356 single irradiation experiments into 36 groups that differ only in terms of ion species, energy, and impact angle. The ice temperatures ranged between $100 \mathrm{~K}$ and $89 \mathrm{~K}$ during the 356 measurements, with the average and standard deviation of $93 \mathrm{~K}$ and $2 \mathrm{~K}$, respectively. Within this limited range, the sputtering yields did not show any significant trend with temperature either.

We first verified if the loss rate from the microbalance during ion irradiation changed with irradiation time. To this end, we kept irradiating the same spot for 5 to 17 minutes with 30 and $50 \mathrm{keV} \mathrm{Ar}{ }^{+}$and $\mathrm{O}_{2}^{+}$beams of $10^{11} \mathrm{~cm}^{-2} \mathrm{~s}^{-1}$. The final sputtering yield $Y$ versus the one derived at the onset of irradiation was found to be $1.10 \pm 0.05$ times higher. The same slight increase of yield with exposure time appeared when we compared the yields derived from experiments with freshly deposited ice films to the yields from all experiments (Table 1). This effect might be due to the ice film becoming more compact under irradiation. Alternatively, it might reflect the buildup of $\mathrm{O}_{2}$ in the ice, which is only released after a certain threshold dose has been exceeded (see Section 4.3). The observed increase in yield would agree with the expected fraction of $\mathrm{O}_{2}$ in the sputtered material (see Section 2). We cannot determine which of the two hypotheses is correct because we lacked a means to independently determine the mass and porosity of the ice film. We use the observed $10 \%$ relative change as an estimate of the uncertainty for a single data point of $Y$ in the subsequent results. The presented sputtering yields will be representative for saturated ice films. Regarding electric properties, the ice films were so thin that the surface did not charge up during ion irradiation. This is an experimental advantage compared to thicker ice layers (Shi et al., 2012, Galli et al., 2016). We also verified that the microbalance did not warm up during irradiation.

205 Another effect beside the dose effect that may affect the accuracy of the derived sputtering yield is the thickness of the ice film. Ideally, the ice film on the microbalance should be much thicker than the average penetration depth of the ions (predicted with SRIM numerical simulations (Ziegler et al., 1985, 2008)). For low ion energies, this can easily be achieved, but for energies much higher than $10 \mathrm{keV}$ the penetration depth is several hundreds of $\mathrm{nm}$, which corresponds to a heavier mass load than the microbalances should be operated at. We therefore checked at low energies if the ice film thickness had any influence on the results. An exemplary plot for one of the 
36 groups $\left(10 \mathrm{keV} \mathrm{O}+\right.$ ions at an impact angle of $\left.45^{\circ}\right)$ is shown in Fig. 1, 215 top row. The red line indicates the simulated penetration depth. For 18 out of 36 groups, measurements both at ice film thicknesses thinner and thicker than the expected ion penetration depth were available. For these cases, $Y$ increased on average by only $1.1 \pm 0.16$ if we averaged over data points obtained at ice films thicker than the expected penetration depth compared with the average over all data points. The only notable effect of film thickness on measured sputtering yields was the increase of scatter for very thin films. The bottom row of Fig. 1 shows a compilation of all 356 single sputtering experiments. Here, the yield rates have been divided by the values found for experiments at thick ice films and the ice film thickness has been normalized to the expected ion penetration depth (red line at 1.0).

We derived the sputtering yield $Y$ for each of the 36 groups of experiments the following way: For the 18 groups where we had measurements at ice films thicker than the expected penetration depth $d+1 \sigma$, we averaged $Y$ over this subset. As uncertainty, we used the standard deviation of $Y$ or the $10 \%$ relative error of single measurements, whichever was larger. For the other 18 groups where no such measurements were available we averaged over the subgroup of data for ice films thicker than $70 \mathrm{~nm}$. The differences between the averages of subgroups and complete groups served as estimates for the uncertainty of $Y$. The resulting 36 values of $Y$ for the different groups are presented in Table 1 . The impact angle $\theta$ is the angle relative to the surface normal as in Equation 1. The energy in Table 1 is the total kinetic energy per ion.

Our sputtering yields agree with the so far existing body of experiments. For the directly comparable ion species $\mathrm{Ar}^{+}$and $\mathrm{O}^{+}$that were also used in the studies serving as input to Eq. 1, the ratio of sputtering yields from the present study and from Famá et al. (2008) vary between 0.7 and 2.9 with an average of 1.6 and a standard deviation of 0.7 . The sputtering yield increases for shallower impact angles $\theta$, although the dependence of $Y$ with $\cos ^{-f}(\theta)$ (see Equation 1) in our experiments is less pronounced $(f=1.0)$ than the averages $f=1.66$ and 1.78 for $\mathrm{O}^{+}$and $\mathrm{Ar}^{+}$observed by Famá et al. (2008).

We then checked with doubly charged $\mathrm{Ar}^{2+}$ and $\mathrm{O}^{2+}$ ions if the charge state of the impacting ion influenced the sputtering yield. Unfortunately, the fluxes of $\mathrm{Ar}^{2+}$ and $\mathrm{O}^{2+}$ ions were low, which resulted in a large uncertainty of yields. The three direct comparisons in Table 1 show yield ratios for doubly versus singly charged ions of $0.5,1.3$, and 1.7 for $50 \mathrm{keV} \mathrm{O}^{n+}$ and $50 \mathrm{keV}$ $\mathrm{Ar}^{n+}$ at $\theta=45^{\circ}$ and $60^{\circ}$. The comparison at same ice film thicknesses yields 

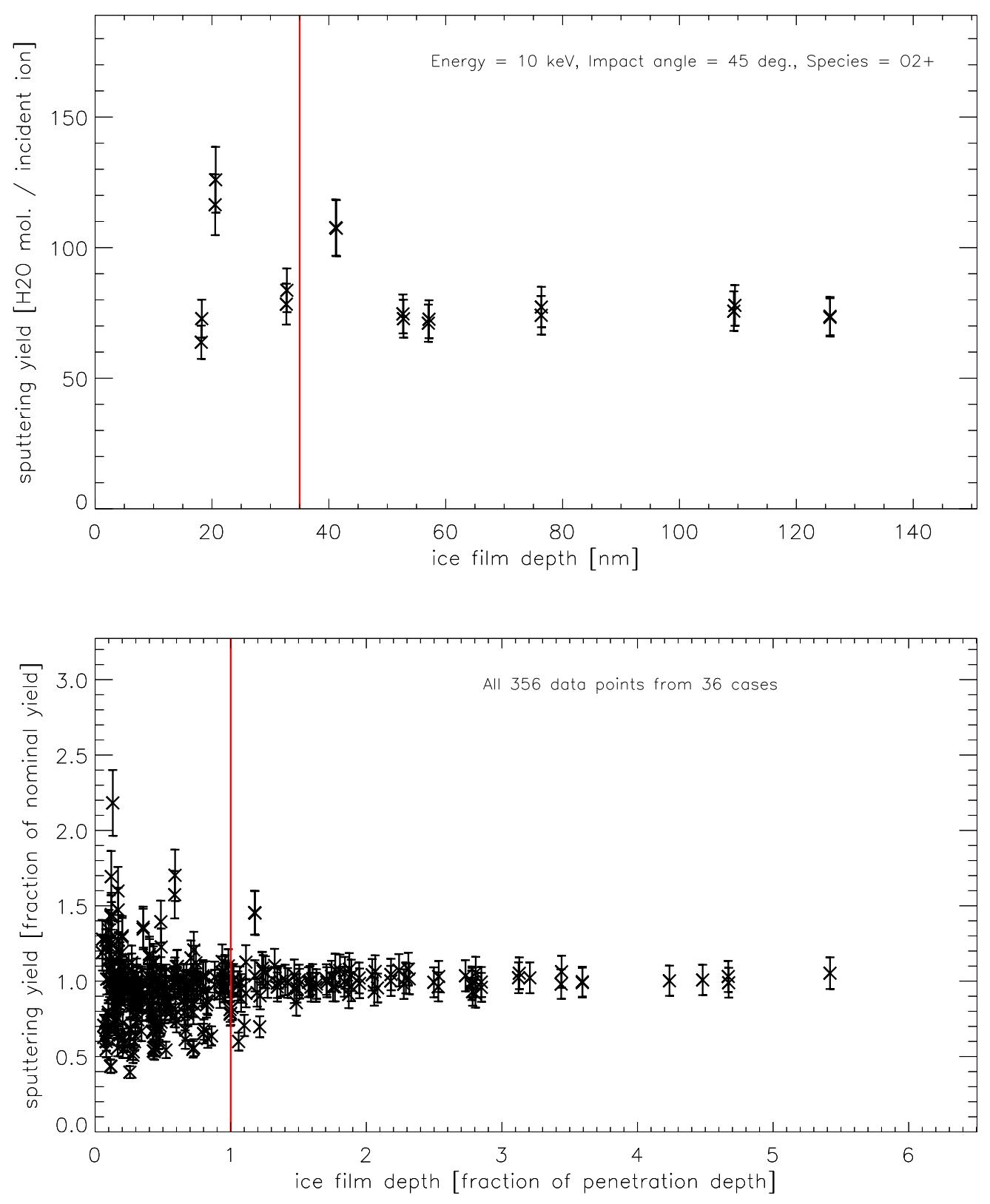

Figure 1: Top: Sputtering yield for $10 \mathrm{keV} \mathrm{O}_{2}^{+}$ions hitting an ice film as a function of ice film thickness. The red line indicates the penetration depth predicted with SRIM. Bottom: All 356 data points in one plot, thickness is scaled to the respective penetration depth and sputtering yield is scaled to the respective value stated in Table1. 
Table 1: Sputtering yields $Y$ for all 36 parameter groups. The expected values $Y_{\exp }$ were calculated with the semi-empirical formula in Eq. 1 for an ice film temperature of $93 \mathrm{~K}$. The $Y_{\exp }$ for $\mathrm{O}_{2}^{+}$was assumed to be twice the value for $\mathrm{O}^{+}$whereas charge state was assumed to not influence $Y_{\exp }$. The penetration depth $d$ and its uncertainty $\sigma_{d}$ (the straggle) were calculated with SRIM.

\begin{tabular}{|c|c|c|c|c|c|c|c|c|}
\hline Group & Ion & Energy $(\mathrm{keV})$ & Angle $\left(^{\circ}\right)$ & $Y$ & $\sigma_{Y}$ & $Y_{\text {exp }}$ & $d(\mathrm{~nm})$ & $\sigma_{d}(\mathrm{~nm})$ \\
\hline 1 & $\mathrm{Ar}^{+}$ & 3 & 45 & 17 & 2 & 24 & 9.6 & 3.9 \\
\hline 2 & $\mathrm{Ar}^{+}$ & 10 & 45 & 51 & 5 & 34 & 21 & 9 \\
\hline 3 & $\mathrm{Ar}^{+}$ & 30 & 45 & 114 & 11 & 48 & 50 & 18 \\
\hline 4 & $\mathrm{Ar}^{+}$ & 50 & 45 & 117 & 12 & 62 & 78 & 27 \\
\hline 5 & $\mathrm{Ar}^{+}$ & 3 & 30 & 13 & 1 & 17 & 11.5 & 4 \\
\hline 6 & $\mathrm{Ar}^{+}$ & 10 & 30 & 37 & 4 & 23 & 25 & 9 \\
\hline 7 & $\mathrm{Ar}^{+}$ & 30 & 30 & 89 & 9 & 33 & 61 & 19 \\
\hline 8 & $\mathrm{Ar}^{+}$ & 50 & 30 & 125 & 13 & 43 & 96 & 28 \\
\hline 9 & $\mathrm{Ar}^{+}$ & 30 & 60 & 150 & 28 & 88 & 36 & 17 \\
\hline 10 & $\mathrm{Ar}^{+}$ & 50 & 60 & 154 & 22 & 114 & 56 & 24 \\
\hline 11 & $\mathrm{Ar}^{2+}$ & 20 & 45 & 96 & 10 & 41 & 35 & 13 \\
\hline 12 & $\mathrm{Ar}^{2+}$ & 50 & 45 & 195 & 41 & 62 & 78 & 27 \\
\hline 13 & $\mathrm{Ar}^{2+}$ & 100 & 45 & 267 & 58 & 104 & 150 & 50 \\
\hline 14 & $\mathrm{Ar}^{2+}$ & 20 & 60 & 73 & 7 & 76 & 25 & 12 \\
\hline 15 & $\mathrm{Ar}^{2+}$ & 50 & 60 & 196 & 20 & 114 & 56 & 24 \\
\hline 16 & $\mathrm{Ar}^{2+}$ & 100 & 60 & 358 & 93 & 193 & 110 & 45 \\
\hline 17 & $\mathrm{O}^{+}$ & 10 & 45 & 48 & 5 & 27 & 35 & 15 \\
\hline 18 & $\mathrm{O}^{+}$ & 30 & 45 & 78 & 11 & 66 & 102 & 40 \\
\hline 19 & $\mathrm{O}^{+}$ & 50 & 45 & 93 & 15 & 107 & 169 & 59 \\
\hline 20 & $\mathrm{O}^{+}$ & 10 & 30 & 44 & 4 & 20 & 43 & 17 \\
\hline 21 & $\mathrm{O}^{+}$ & 30 & 30 & 64 & 9 & 47 & 123 & 42 \\
\hline 22 & $\mathrm{O}^{+}$ & 50 & 30 & 74 & 7 & 77 & 207 & 63 \\
\hline 23 & $\mathrm{O}^{2+}$ & 50 & 45 & 46 & 6 & 66 & 169 & 59 \\
\hline 24 & $\mathrm{O}^{2+}$ & 80 & 45 & 45 & 17 & 186 & 270 & 85 \\
\hline 25 & $\mathrm{O}_{2}^{+}$ & 3 & 45 & 22 & 2 & 34 & 13 & 6 \\
\hline 26 & $\mathrm{O}_{2}^{+}$ & 10 & 45 & 74 & 7 & 52 & 35 & 15 \\
\hline 27 & $\mathrm{O}_{2}^{+}$ & 20 & 45 & 159 & 22 & 88 & 70 & 27 \\
\hline 28 & $\mathrm{O}_{2}^{+}$ & 30 & 45 & 165 & 23 & 125 & 102 & 40 \\
\hline 29 & $\mathrm{O}_{2}^{+}$ & 40 & 45 & 186 & 26 & 168 & 133 & 50 \\
\hline 30 & $\mathrm{O}_{2}^{+}$ & 50 & 45 & 200 & 28 & 214 & 169 & 59 \\
\hline 31 & $\mathrm{O}_{2}^{+}$ & 10 & 30 & 66 & 7 & 40 & 43 & 17 \\
\hline 32 & $\mathrm{O}_{2}^{+}$ & 30 & 30 & 128 & 18 & 89 & 123 & 42 \\
\hline 33 & $\mathrm{O}_{2}^{+}$ & 50 & 30 & 155 & 22 & 220 & 207 & 63 \\
\hline 34 & $\mathrm{O}_{2}^{+}$ & 10 & 60 & 83 & 8 & 98 & 27 & 14 \\
\hline 35 & $\mathrm{O}_{2}^{+}$ & 30 & 60 & 182 & 18 & 224 & 74 & 34 \\
\hline 36 & $\mathrm{O}_{2}^{+}$ & 50 & 100 & 264 & 37 & 382 & 124 & 55 \\
\hline
\end{tabular}


values of 46 vs. 73,165 vs. 154 , and 163 vs. 106. We conclude from these measurements that the charge state has no notable effect on the sputtering yield from water ice films for $50 \mathrm{keV}$ ions. This agrees with recent experiments presented by Muntean et al. (2015, 2016) for doubly charged C, N, and O ions irradiating $\mathrm{O}_{2}$ and $\mathrm{H}_{2} \mathrm{O}$ ice films at $4 \mathrm{keV}$. No experimental evidence exists so far that highly charged ions sputter more water ice than singly charged ions do. This so-called potential sputtering effect was usually observed for targets like $\mathrm{LiF}, \mathrm{NaCl}, \mathrm{SiO}_{2}$ and $\mathrm{Al}_{2} \mathrm{O}_{3}$ with a strong electron-phonon coupling, but did not occur in $\mathrm{Au}, \mathrm{Si}$, and GaAs for example (Aumayr and Winter, 2004).

The sputtering yield for molecular $\mathrm{O}_{2}^{+}$is an interesting result as it deviates from expectations. From Table 1 we derive empirically that $Y_{\mathrm{O}_{2}}=1.1 \times Y_{\mathrm{O}}$ at $3 \mathrm{keV}, Y_{\mathrm{O}_{2}}=1.5 \times Y_{\mathrm{O}}$ at $10 \mathrm{keV}$, and $Y_{\mathrm{O}_{2}}=(2.1 \pm 0.1) \times Y_{\mathrm{O}}$ at 30 and $50 \mathrm{keV}$. The same ratios are found for two different impact angles. Figure 2 illustrates the $\mathrm{O}^{+}$and $\mathrm{O}_{2}^{+}$sputtering yields measured at an impact angle of $45^{\circ}$. The data point for $3 \mathrm{keV} \mathrm{O}^{+}$was obtained at ice temperatures different from the other measurements $(124 \mathrm{~K})$; it was scaled to average temperature for comparison's sake but was omitted from Table 11. The data points in Fig. 2 are compared to the prediction for $Y_{\mathrm{O}}(E)$ from Equation 1 (solid blue line). To interpret the $\mathrm{O}_{2}^{+}$measurements, we added the predictions for $2 Y_{\mathrm{O}}(E / 2)$ (dotted blue line) and $4 Y_{\mathrm{O}}(E / 2)$ (dashed-dotted red line).

In the single-collision regime, the experimental yields of $\mathrm{O}^{+}$and $\mathrm{O}_{2}^{+}$are indistinguishable. Our conjecture is that the total kinetic energy dominates the sputtering outcome at these low energies. For higher energies, one would naively expect that a molecule fragments upon impact and then triggers sputtering via its constituents, with the total kinetic energy $E$ equally distributed among the fragments:

$$
Y_{\mathrm{O}_{2}}(E)=2 Y_{\mathrm{O}}\left(\frac{E}{2}\right) \text {. }
$$

If Equation 4 were true for the electronic sputtering regime, then $Y_{\mathrm{O}_{2}}(E) \approx$ $Y_{\mathrm{O}}(E)$ for 30 and $50 \mathrm{keV}$ (Equation 1). The observed sputtering yield, however, is two times higher. A similar result was obtained for a thick and porous ice layer irradiated with $30 \mathrm{keV} \mathrm{O}^{+}$and $\mathrm{O}_{2}^{+}$ions (Galli et al., 2015). Equation 1 for a heavy nucleus of mass 32 and $Z=16$ would also match the observed sputtering yield at $3 \mathrm{keV}$. For energies above $10 \mathrm{keV}$, on the other hand, the yield would be even smaller than for $\mathrm{O}^{+}$. We do not show this yield curve because it is conceptually wrong anyway to interpret a molecule as a large nucleus. Equation 1, which was derived for nuclei only, must be 
expanded in a general way to also accommodate molecular sputtering. We suspect that cooperative effects between the two oxygen atoms in the $\mathrm{O}_{2}^{+}$are responsible for the increased sputtering yield. To test this interpretation we plan for additional sputtering experiments with other molecules in the near future.

\subsection{Double set-up: opening angle of the sputtering cone}

To create a strong enough signal of accreted water molecules on the secondary microbalance, we employed the heaviest ion $\left(\mathrm{O}_{2}^{+}\right)$. Even so, only 8 out of these 32 sputtering measurements produced a signal that could be discriminated on the secondary microbalance against the noise. Each of the 32 irradiations lasted one or two minutes with a beam current of roughly 2 $\mathrm{nA}$ on the primary microbalance. The chamber pressure was $10^{-8} \mathrm{mbar}$ and the ice film thickness on the secondary microbalance ranged between 20 and $65 \mathrm{~nm}$. Frequency differences of at least $1 \mathrm{mHz} \mathrm{s}^{-1}$ were required to create a detectable signal. The clearest example of such a detection is shown in Figure 3. The immediate drop and rise in frequency at the beginning and the end of irradiation are related to the energy deposited by the ion beam. Taking into account all detections, the mean ratio of secondary accretion rate to primary loss rate was $0.015 \pm 0.005$ for different impact angles $45^{\circ}$ to $60^{\circ}$ and for the three studied energies 10,30 , and $50 \mathrm{keV}$. The active surface $\left(0.316 \mathrm{~cm}^{2}\right)$ of the secondary microbalance was always located $2.5 \mathrm{~cm}$ above the irradiated ice film. As expected, no significant $(2 \sigma)$ pressure rises were ever observed in the chamber during irradiation. Most of the ejected particles stuck to the cold surfaces of the opposite microbalance.

The ratio of 0.015 agrees with expectations from previous experiments for ejection angle and sticking probability: Gibson et al. (2011) reported a sticking probability of $0.98 \pm 0.03$ for suprathermal water molecules (0 to 1 $\mathrm{eV}$ ) impacting ice films ( $T$ between 110 and $155 \mathrm{~K}$ ) at a variety of impact angles for crystalline and for amorphous ice. In the experiments performed by (Vidal et al., 2005), 2/3 of all sputtered water molecules were ejected within a $40^{\circ}$ angle. If we assume for simplicity's sake a sticking probability of 1 and further assume that all ejecta were uniformly distributed within a $45^{\circ}$ cone relative to the surface normal independent of the ion impact angle, the active surface of the microbalance opposite to the irradiated ice really sampled a fraction of $0.3 \mathrm{~cm}^{2} / 20 \mathrm{~cm}^{2}=0.015$.

The secondary microbalance could be used to derive a sputtering yield when the target ice sample can no longer be deposited onto a microbalance. 


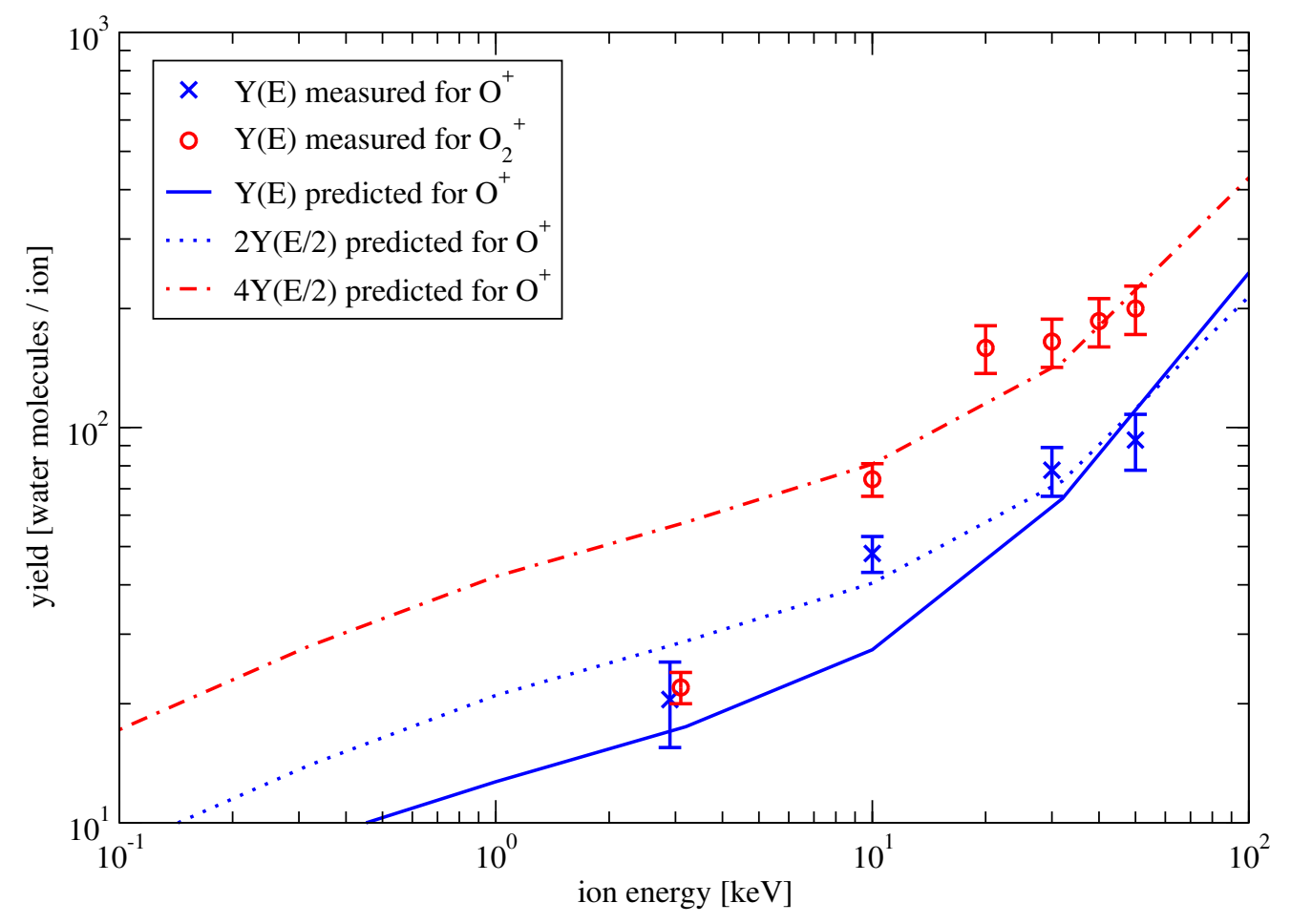

Figure 2: $\mathrm{O}^{+}$and $\mathrm{O}_{2}^{+}$sputtering yields versus energy. Data points are taken from the present study, the solid $(Y(E))$, dotted $(2 Y(E / 2))$, and dashed-dotted $(4 Y(E / 2))$ lines are predictions for $\mathrm{O}^{+}$based on previous ice sputtering experiments (Famá et al., 2008). At $10 \mathrm{keV}$, the prediction seems to underestimate the observed $\mathrm{O}^{+}$sputtering yield. For energies above $10 \mathrm{keV}, 4 Y(E / 2)$ matches the observed $\mathrm{O}_{2}^{+}$sputtering yield better than the expected $2 Y(E / 2)$ does. 


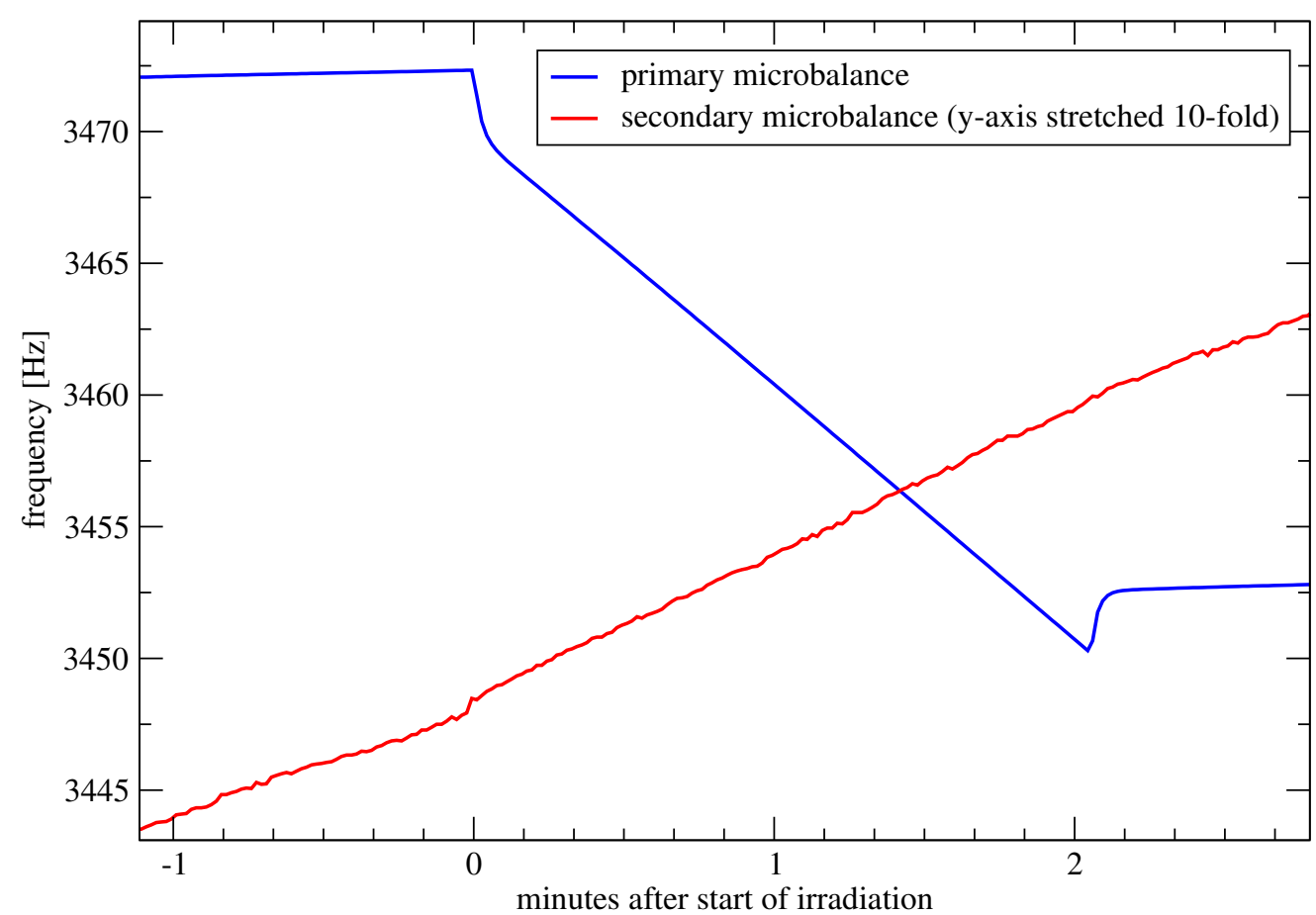

Figure 3: Frequency signals for double microbalance set-up. The primary microbalance (blue curve) shows a clear signal when a $50 \mathrm{keV} \mathrm{O}+$ ion beam hits the surface at time zero. The secondary signal of accreted water ice on the microbalance opposite to it is much weaker (red curve, stretched by a factor of 10 in y-direction). 
The difficulties of such an indirect measurement will be discussed in more 325 detail in Section 4.4. Relevant for that discussion is the observed variability of secondary accretion rate to primary loss rate. This variability implies a $33 \%$ uncertainty with which one could derive absolute sputtering yields with a secondary microbalance. Additional assumptions one would have to make are that the sticking probability and the opening cone do not change with ion species and impact angles.

\subsection{Electron irradiation of ice films}

Beside the ion experiments presented in Sections 4.1 and 4.2, we also irradiated the water ice films with electron beams of $0.1,1$, and $3 \mathrm{keV}$ and beam intensities of $(6-60) \times 10^{12} \mathrm{e}^{-} \mathrm{s}^{-1} \mathrm{~cm}^{-2}$. As for the case of ions, the ice film thickness must be compared with the penetration depth $d$ : it calculates to $d=0.8 \mathrm{~nm}$ for $100 \mathrm{eV}$ electrons, whereas for $1 \mathrm{keV} d=46 \mathrm{~nm}$ and for 10 $\mathrm{keV}$ electrons $d=3 \mu \mathrm{m}$ (Equation 3). During our experiments, the ice films were between 20 and $85 \mathrm{~nm}$ thick. Thus, the 0.1 and $1 \mathrm{keV}$ electron beams deposited all energy inside the ice.

Figure 4 shows an example of a $0.1 \mathrm{keV}$ electron beam with $6 \times 10^{12} \mathrm{e}^{-}$ $\mathrm{s}^{-1} \mathrm{~cm}^{-2}$ irradiating a freshly deposited ice film on the microbalance during 10 minutes. The frequency drops immediately due to the momentum of the electrons and the frequency rate turns negative, indicating ice is being eroded. However, a constant loss rate is achieved only 5 minutes after the onset of irradiation. This evolution with time cannot be explained by varying beam strength - the frequency glitches at the beginning and the end are equal within 10\%. Rather it shows that a minimum dose of $2 \times 10^{15} \mathrm{e}^{-} \mathrm{cm}^{-2}$ is required until a constant loss rate is achieved. This figure agrees well with the dose dependence of the electron-stimulated production and release of $\mathrm{O}_{2}$ derived by Orlando and Sieger (2003) for $\mathrm{D}_{2} \mathrm{O}$ ice films at $120 \mathrm{~K}$. For subsequent electron irradiation at higher energies, the frequency rate changed immediately to a constant loss rate. This also shows that, as for the case of ion irradiation, the ice film does not charge up to any potential that could deflect the beam. When irradiation of a fresh ice film is interrupted after less than 5 minutes and recommenced two minutes afterwards, the frequency rate continues at the rate it had at the end of the previous irradiation. This indicates that electron irradiation permanently alters the ice film, as noticed previously by Reimann et al. (1984); Johnson et al. (2003). The energy of the 1 and $3 \mathrm{keV}$ electron beams warm up the microbalance by a small but noticeable amount of at most $0.5 \mathrm{~K}$. Since these temperature changes lag 


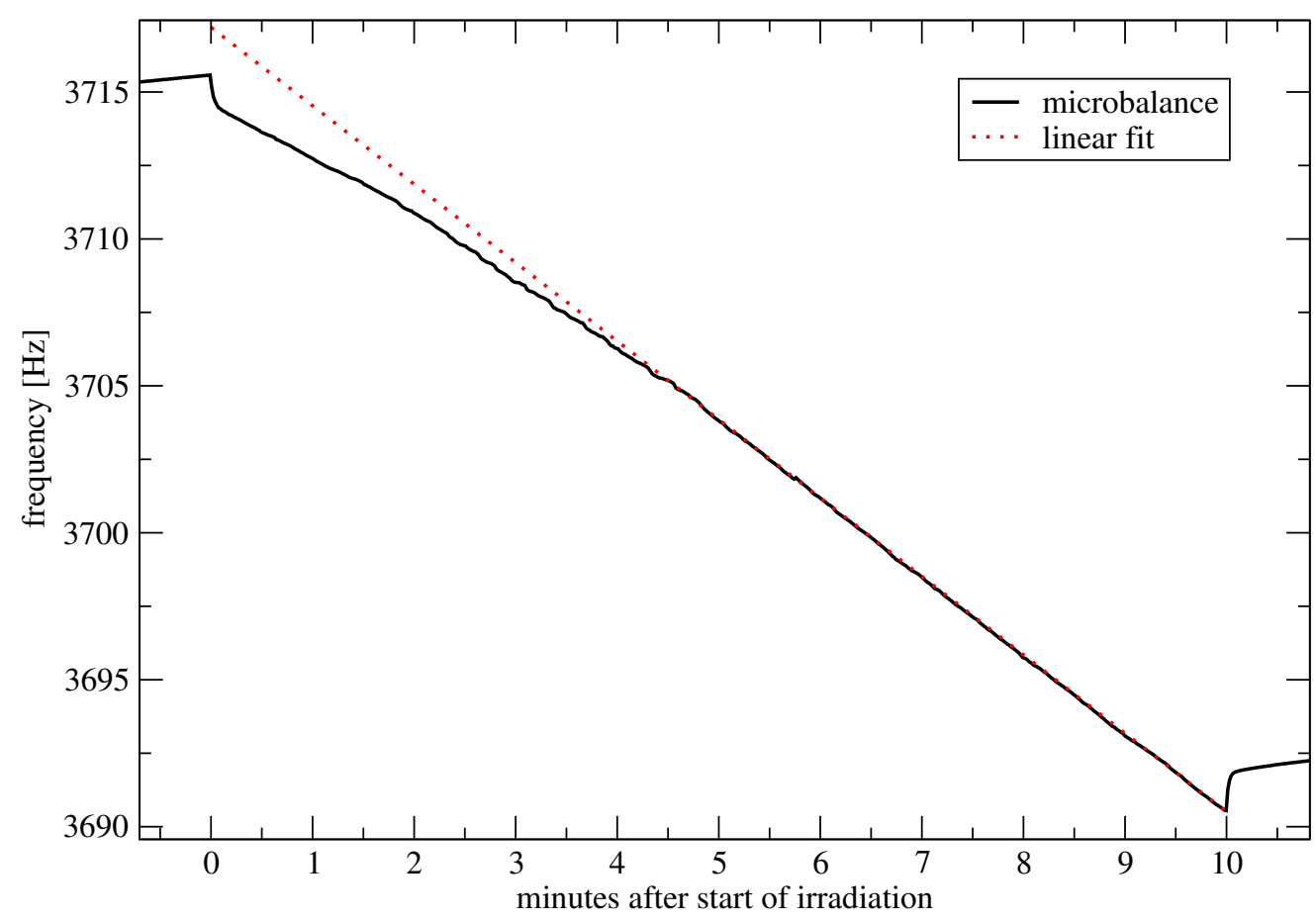

Figure 4: Mass loss from microbalance upon irradiation with a $0.1 \mathrm{keV}$ electron beam. Five minutes after the start a constant loss rate is observed, which does not change any more until the electron beam is shut down 10 minutes after the start.

behind the start and the stop of irradiation by $5 \pm 1$ minutes, sublimation cannot explain the mass loss indicated by the microbalance frequency.

We also tested the sputtering yield for $30 \mathrm{keV} \mathrm{O}_{2}^{+}$ions after 30 minutes of electron irradiation with a deposited dose of 1,3 , and $5 \times 10^{16} \mathrm{e}^{-} \mathrm{cm}^{-2}$ : within a factor of 2 the sputtering yields were the same as the values in Table 1 for ice films prior to electron irradiation.

Upon irradiation, the frequency of the microbalance indicated a mass loss, which we then translated into an $\mathrm{H}_{2} \mathrm{O}$ sputtering yield in analogy to the case for ions. Since water is rather released in the form of $\mathrm{H}_{2}$ and $\mathrm{O}_{2}$ than of $\mathrm{H}_{2} \mathrm{O}$, 370 we use the term "water equivalent yield" when we quantify sputtering yield from electrons. The results of these experiments are summarized in Table 2. The main factor limiting the accuracy is the difficulty of keeping the electron 
Table 2: Measured and expected electron sputtering yields for water equivalent mass loss per incident electron. $\theta$ denotes the impact angle relative to the surface normal and $T$ is the ice temperature.

$\begin{array}{rrrrrr}\text { Energy }(\mathrm{keV}) & \theta\left(^{\circ}\right) & T(\mathrm{~K}) & Y \pm \sigma_{Y} & Y_{\exp } & d(\mathrm{~nm}) \\ 0.1 & 45^{\circ} & 93 & 0.8 \pm 0.4 & 1.5 & 0.8 \\ 1.0 & 45^{\circ} & 91 & 1.9 \pm 0.6 & 1.5 & 46 \\ 3.0 & 45^{\circ} & 91 & 2.3 \pm 0.8 & 0.65 & 320\end{array}$

beam current stable and monitor it over several minutes on a small area $\left(0.316 \mathrm{~cm}^{2}\right)$ compared to the entire beam size of several $\mathrm{cm}$. Nevertheless, we find a water equivalent yield on the order of unity. This is the same order of magnitude as the estimate $Y=0.3 \pm 0.15$ in Galli et al. (2016). There, we irradiated deep porous ice layers and frost covered metal at $T=116 \mathrm{~K}$ with very wide 50 and $100 \mathrm{eV}$ electron beams. The predicted water equivalent yield rates in Table 2 were derived from the $\mathrm{O}_{2}$ yield in Equation 2 under the assumption that per $\mathrm{O}_{2}$ molecule two water molecules are lost from the microbalance. For 0.1 and $1 \mathrm{keV}$, the predicted values agree within a factor of 2 with our results, which we consider acceptable at the given uncertainties. For $3 \mathrm{keV}$, Equation 2 underestimates the true production threefold. The experimental yield for $3 \mathrm{keV}$ may not be the definite answer yet as the ice film thickness was necessarily much thinner than the relevant penetration depth. To properly study the yield rate for electron energies much larger than 1 $\mathrm{keV}$, we must resort to deep $(\simeq \mathrm{mm})$ ice samples, which poses new challenges regarding the detection method (see Section 4.4). In future experiments we will also direct narrower electron beams at a variety of icy targets to see if the sputtering yield and chemical reactions depend on the physical properties of the ice.

The total pressure rise in the chamber due to electron irradiation was on the order of $10^{-9}$ mbar (see Section 4.4). This increase allowed us to identify the most abundant species with partial pressures above $10^{-10}$ mbar in the mass spectrometer. The pressure rises due to ions, on the other hand, amounted only to several $10^{-11}$ mbar because of the much lower beam intensity. The ion sputtering signal therefore could not be analysed with the mass spectrometer. For an electron beam intensity of $(6-60) \times 10^{12} \mathrm{e}^{-} \mathrm{s}^{-1} \mathrm{~cm}^{-2}$ and an irradiation duration of 2 to 10 minutes we found the relative chemical abundances as specified in Table 3. For electrons with a penetration depth 
Table 3: Chemical composition per volume of released gas during electron irradiation of water ice films. $\mathrm{H}$ and $\mathrm{O}$ are fragmentation products of $\mathrm{H}_{2}$ and $\mathrm{O}_{2}$.

\begin{tabular}{rrrrrr}
\hline Energy (keV) & $\mathrm{H} \mathrm{\&} \mathrm{H}_{2}$ & $\mathrm{O} \mathrm{\&} \mathrm{O}_{2}$ & $\mathrm{HO} \& \mathrm{H}_{2} \mathrm{O}$ & $\mathrm{H}_{3} \mathrm{O}$ & Other species \\
0.1 & $30 \pm 10 \%$ & $30 \pm 10 \%$ & $5 \pm 5 \%$ & $<5 \%$ & $35 \pm 10 \%$ \\
1.0 & $25 \pm 5 \%$ & $20 \pm 10 \%$ & $5 \pm 5 \%$ & $<5 \%$ & $50 \pm 10 \%$ \\
3.0 & $20 \pm 5 \%$ & $11 \pm 5 \%$ & $2 \pm 2 \%$ & $<2 \%$ & $67 \pm 10 \%$
\end{tabular}

less than the ice film thickness $(55 \pm 10 \mathrm{~nm})$, at least half of the observed pressure rise was due to water-related compounds $\left(\mathrm{H}, \mathrm{H}_{2}, \mathrm{O}, \mathrm{O}_{2}, \mathrm{HO}, \mathrm{H}_{2} \mathrm{O}\right.$, and $\mathrm{H}_{3} \mathrm{O}$ ). For the $3 \mathrm{keV}$ electron beam, this ratio dropped to $33 \%$. This may be due to the insufficient ice film thickness for high energies. Water was lost from the ice film predominantly as $\mathrm{H}_{2}$ and $\mathrm{O}_{2}$, whereas $\mathrm{H}_{2} \mathrm{O}$ partial pressures never rose significantly above the base pressure. Most, if not all, atomic $\mathrm{H}$ and $\mathrm{O}$ had been fragmented from molecular $\mathrm{H}_{2}$ and $\mathrm{O}_{2}$ in the mass spectrometer. The composition of the non-water species depended on the main residual gases already present before electron irradiation $\left(\mathrm{N}_{2}\right.$ and $\mathrm{CO}_{2}$ for instance). For comparison's sake, we also directed the $3 \mathrm{keV}$ electron beam at warm metal surfaces and found a total pressure rise two times lower, and no unambiguously water-related species, than when we targeted the ice-covered microbalance. This signal must be caused by contaminants on the targeted metal and possibly by other contaminants on the walls that were detached from the walls by secondary electrons. We plan for more accurate and extensive examinations of the irradiation products with mass spectrometry in the near future. In the meanwhile, we recommend for the water-related components due to electron irradiation $Y_{\mathrm{H}_{2} \mathrm{O}}=(0.1 \pm 0.1) Y, Y_{\mathrm{H}_{2}}=(0.6 \pm 0.1) Y$, and $Y_{\mathrm{O}_{2}}=(0.3 \pm 0.1) Y$ where $Y$ is the energy-dependent sputtering yield in Table [2].

\subsection{The correlation of chamber pressure with sputtering yield}

For the single microbalance set-up, we evaluated how well the observed pressure rise due to the sputtering signal correlated with the observed mass loss from the microbalance. This is important if we want to use the measured gas pressure in the chamber as a proxy for sputtering rates from thick ice samples that cannot be attached on the tip of a microbalance. The other indirect approach would be to mount a secondary microbalance above the target (see Section 4.2).

We adapted the equation from Galli et al. (2016), 


$$
\Delta p_{p}=Y \frac{i}{e^{-}} \frac{k_{B} T}{S}
$$

430

to convert the amount of sputtered molecules $Y i / e^{-}$derived with the microbalance (see Table 1 into an expected pressure rise $\Delta p_{p}$. This was to be compared to the actually measured pressure rise $\Delta p$. The pumping speed $S$ was determined from the time scale at which a pressure signal receded to background levels once the source was switched off. It was found to be $S=0.35 \pm 0.1 \mathrm{~m}^{3} \mathrm{~s}^{-1}$ for water vapour as well as for $\mathrm{O}_{2}$ and $\mathrm{N}_{2}$ in the chamber. $T \approx 300 \mathrm{~K}$ is the temperature of the chamber walls, and $i$ is the current of all ions or electrons that irradiate the ice-covered surface.

We selected all ion sputtering experiments during which the residual pressure was low enough (below $10^{-8}$ mbar) that the sputtering signal exceeded the detection threshold of the pressure gauge. The experiments meeting these requirements were performed with 30 and $50 \mathrm{keV} \mathrm{O}+$ beams of $\sim 1 \mathrm{nA}$. For electron sputtering, no selection was necessary because the beam current, and thus the pressure rise, was orders of magnitude stronger. In total, 29 ion experiments and 15 electron experiments were available for this comparison. We assumed that all eroded mass was released as $\mathrm{H}_{2} \mathrm{O}$ into the chamber in the case of ion sputtering. In the case of electron irradiation, most water molecules were released as $\mathrm{H}_{2}+\frac{1}{2} \mathrm{O}_{2}$ (see Table 3). On the other hand, the electron beam was spatially more extended resulting in $50 \%$ of the electrons hitting warm metal surfaces instead of water ice. Considering the results in Section 4.3 we therefore treated electron irradiation the same way as ion irradiation: we multiplied all emitted electrons with the water equivalent yield rate from Table 2 ,

Figure 5 shows the measured versus the predicted pressure rise $\Delta p_{p}$ in the chamber. Taking into account all signals whose $\Delta p>1.5$ times the detection threshold of $10^{-11}$ mbar, the average conversion ratio calculates to $0.10 \pm 0.03$. The uncertainty is the standard deviation, depicted as thin red lines above and below the average ratio in Fig. 5 . The ratio of 0.1 between expected and measured pressure rise is constant throughout the experiments (spanning 5 weeks of experiments over 4 months) and does not vary with signal strength or species of ejecta. The observed pressure rise thus can be converted into a relative sputtering yield over a wide range of pressures and for different sources. The pressure rise method has the same accuracy of $\sim 30 \%$ as the double microbalance method (Section 4.2 ) provided the signals lie well above the detection limit. The apparent conversion ratio for experiments with a 


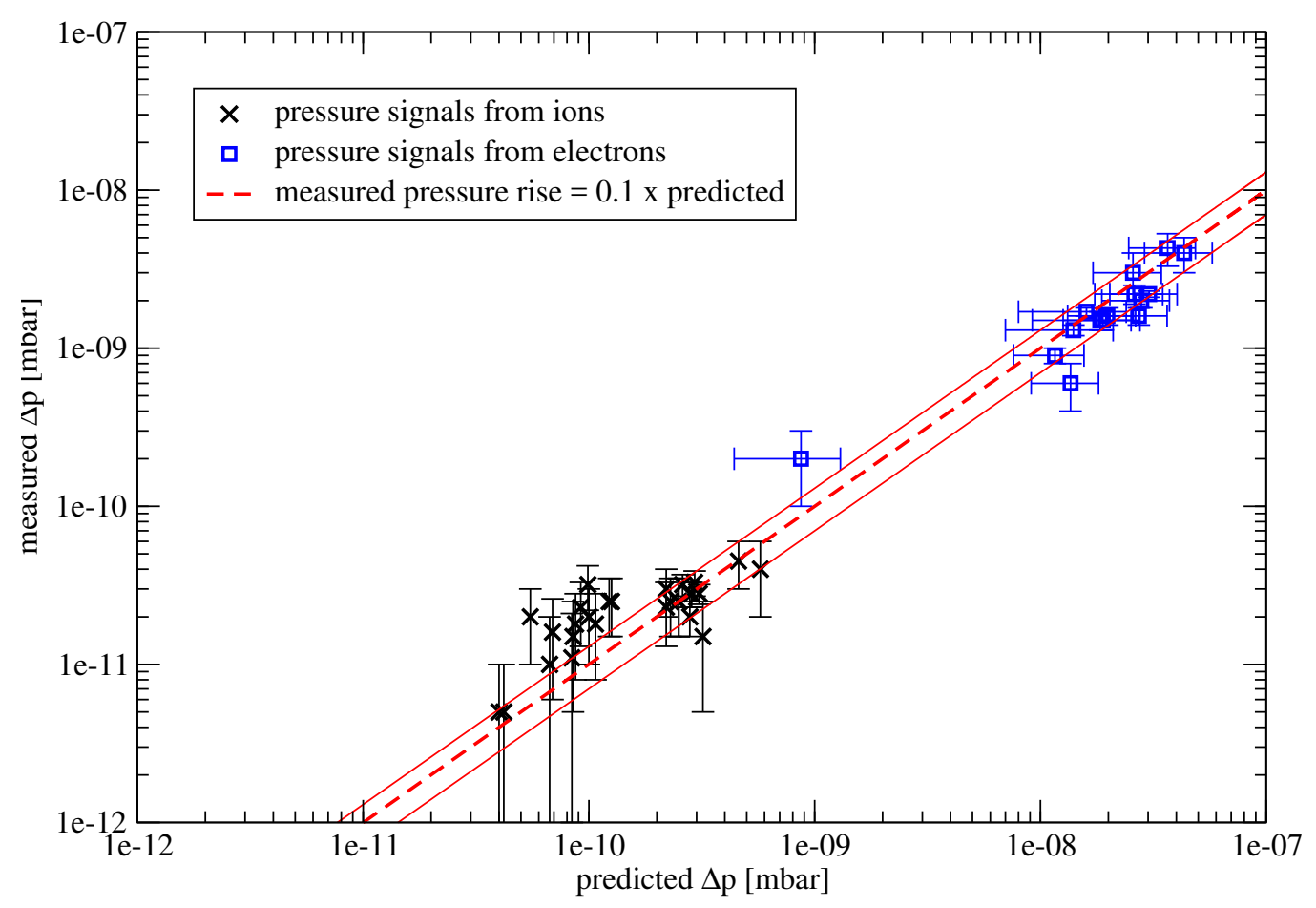

Figure 5: Measured versus expected pressure rise during irradiation of ice films with electron and ion beams.

measured pressure rise close to the detection limit was two times higher (cluster of data to the left in Fig. 5). This confirms the estimate by Galli et al. (2016) of a factor of two uncertainty for the sputtering yields as the signals reported there were close to the detection limit.

The conversion ratio between expected and real pressure depends on experimental conditions, such as pumping speed, chamber volume, chamber surface area, and position of pressure gauge relative to particle source. The ratio also corrects for any shortcomings in the absolute calibration of the pressure gauge itself. The conversion ratio was close to unity for the ion sputtering experiments with thick regolith ice performed in MEFISTO in 475 2015 Galli et al. (2016). After 2015, a large hexapod table and a cooling shroud covering all walls were reintroduced into the chamber. These additional structures increased the macroscopic surface area fourfold. The inner 
shroud surface is sand-blasted to increase the microscopic surface area even more. Moreover, the cooling shroud partially blocked the field-of-view of the pressure gauge. It is therefore vital to calibrate the pressure method in absolute terms for a new experiment set-up. Monitoring any pressure variation of known absolute intensity will do; in this work we used microbalances.

Comparing the pressure rise method with the double-microbalance setup, we find that both methods have a relative accuracy of roughly $30 \%$. Both methods must initially be calibrated in an absolute sense because the fraction of ejecta that actually make it to the pressure gauge or to the secondary microbalance may be much smaller than unity. The pressure rise method seems preferable in terms of sensitivity: Even for the strongest sputtering signals, we detected an accretion rate on the secondary microbalance only in 8 out of 32 cases. If the primary target were replaced by a deep ice sample as described in Galli et al. (2016), the water vapour would be more abundant in the chamber. This would make the frequency of the secondary microbalance noisier, further complicating any detection of the secondary signal caused by sputtering. The pressure rise method, on the other hand, detected a signal in 14 out of 15 cases for the same range of expected pressure rises of $(1-4) \times 10^{-10}$ mbar. A base pressure below $10^{-8}$ mbar for $\mathrm{H}_{2} \mathrm{O}$ was required for both methods. To enhance sensitivity, the pressure gauge would have to be closer to the region of sputtering and with a direct line-of-sight or the distance between microbalance and ice surface would have to be reduced.

On the other hand, the secondary microbalance set-up is better to constrain the spatial distribution of ejecta. For a wider range of experiment parameters and better detection probabilities, the secondary microbalance should be operated with a cooling cycle independent from the primary target. For irradiation experiments where a large fraction of water reacts to $\mathrm{H}_{2}$ and $\mathrm{O}_{2}$, the catcher microbalance would have to be sufficiently cold to collect $\mathrm{O}_{2}$ as well. This did not apply to our ion sputtering experiments because most ejecta were water molecules at the given ice temperatures (see Section 2). For metal or silicate targets that require no cooling (Berger et al., 2017), the approach with a secondary microbalance is easier to implement.

\section{Conclusions}

We have confirmed previous sputtering experiments for $\mathrm{O}^{+}$and $\mathrm{Ar}^{+}$irradiating ice films (Famá et al., 2008; Johnson et al., 2009; Cassidy et al., 2013) at shallow impact angles between $30^{\circ}$ and $60^{\circ}$. 
The ion sputtering yield does not notably increase for doubly charged versus singly charged ions at $50 \mathrm{keV}$. This result challenges the alternative interpretation by Shemansky et al. (2014) of Europa's atmosphere. Their interpretation relied on the hypothesis that the sputtering yield of the icy surface should markedly increase with the charge state of ions. Additional experiments with multiply charged ions at low energy will be needed to decide if potential sputtering occurs in water ice.

Our experiments with molecular oxygen ions imply that $Y\left(\mathrm{O}_{2}^{+}\right) \approx Y\left(\mathrm{O}^{+}\right)$ in the single collision regime. In contrast, the sputtering yield due to molecular oxygen is two times higher than expected in the electronic sputtering regime at 30 and $50 \mathrm{keV}$. A general theory of the sputtering yield due to molecules is beyond the scope of this paper, but the present results are a useful input for atmosphere models of Europa and Ganymede where $\mathrm{O}_{2}$ is expected to be one of the major atmospheric constituents (Shematovich et al. 2005; Marconi, 2007; Plainaki et al., 2012, 2015; Dols et al., 2016). Although oxygen in the Jovian plasma generally is $\mathrm{O}^{+}$(Paranicas et al., 2002), secondary erosion due to molecular oxygen may play an important role because of a cascade of charge-exchanges of $\mathrm{O}_{2}^{+}$ions with $\mathrm{O}_{2}$ in Europa's atmosphere (Dols et al., 2016). This secondary erosion can be quantified in future models with the results presented here.

The time scales and sputtering yields for irradiation with $100 \mathrm{eV}$ electrons agree with previous studies; the ejecta are predominantly $\mathrm{H}_{2}$ and $\mathrm{O}_{2}$. In addition, we obtained the first experimental sputtering yields for electrons around $1 \mathrm{keV}$ on water ice. The results show that the yield levels off at these electron energies. This has major implications for the surface erosion and atmospheres of icy celestial bodies. If a large fraction of ions in the plasma surrounding these objects have energies much higher than $1 \mathrm{keV}$ (as is the case in the magnetospheres of Jupiter (Paranicas et al., 2002) and Saturn (Sergis et al., 2009)), the $\mathrm{H}_{2} \mathrm{O}$ and $\mathrm{O}_{2}$ production due to ions will always dominate over the production rate due to electrons.

Both the pressure rise method and the double microbalance method can be used to measure the sputtering yield of an icy sample not attached to a microbalance. However, the detection thresholds make it difficult to analyse experiments with a low yield. We will prefer the pressure rise method in future experiments since it showed a better sensitivity in this study. Implementing the secondary microbalance approach for deep ice layers over a wide range of parameters also poses an engineering challenge as two independent cooling cycles are required. Both measurement methods introduce a relative 
uncertainty of $30 \%$ to the derived sputtering yield. This accuracy should be sufficient to test if a deep porous ice layer has a $70 \%$ lower sputtering yield compared to a compact ice layer (Cassidy and Johnson, 2005).

555

\section{Acknowledgements}

The work in this paper has been partially performed in the context of the activities of the ISSI International Team Nr. 322, www.issibern. ch/teams/exospherejuice/. We also would like to thank G. Bodmer, J. Gonseth, and A. Etter for their relentless support of the scientific work at the

\section{References}

Aumayr, F. and Winter, H., 2004. Potential sputtering. Philosophical Transactions of the Royal Society A 362, 77-102.

Baragiola, R.A., Atteberry, C.L., Dukes, C.A., Famá, M., Teolis, B.D., 2002. Atomic collisions in solids: Astronomical applications. Nuclear Instruments \& Methods in Physics Research B, 193, 720-726.

Berger, B.M., Szabo, P.S., Stadlmayr, R, Aumayr, F., 2017. Sputtering measurements using a quartz crystal microbalance as a catcher Nuclear Instruments and Methods in Physical Research B, in press.

Boring, J. W., Johnson, R.E., Reimann, C.T., Garret, J.W., Brown, W.L., Marcantonio, K.J., 1983. Ion-induced chemistry in condensed gas solids, Nuclear Instruments \& Methods in Physics Research, 218, 707-711.

Calvin, W.M., Clark, R.N., Brown, R.H., Spencer, J.R., 1995. Spectra of the icy Galilean satellites from 0.2 to $5 \mu \mathrm{m}$ : A compilation, new observations,

Cassidy, T.A., Johnson R.E., 2005. Monte Carlo model of sputtering and other ejection processes within a regolith. Icarus 176, 499-507.

Cassidy, T., Coll, P., Raulin, F., Carlson, R.W., Johnson, R.E., Loeffler, M.J., Hand, K.P., Baragiola, R.A., 2010. Radiolysis and Photolysis of Icy Satellite Surfaces: Experiments and Theory. Space Science Reviews. doi:10.1007/s11214-009-9625-3. 
Cassidy, T.A., Paranicas, C.P., Shirley, J.H., DaltonIII, J.B., Teolis, B.D., Johnson, R.E., Kamp, L., Hendrix, A.R., 2013. Magnetospheric ion sputtering and water ice grain size at Europa. Planetary and Space Science 77, 64-73.

Dols, V.J., Bagenal, F., Cassidy, T.A., Crary, F.J., Delamere, P.A., 2016. Europa's atmospheric neutral escape: Importance of symmetrical $\mathrm{O}_{2}$ charge exchange. Icarus 264, 387-397.

Domingue, D.L., Verbiscer, A., 1997. Re-analysis of the solar phase curves of the icy Galilean satellites. Icarus 128, 49-74.

Famá, M., Shi, J., Baragiola, R.A., 2008. Sputtering of ice by low-energy ions. Surface Science 602, 156.

Galli, A., Pommerol, A., Wurz, P., Jost, B., Scheer, J.A., Vorburger, A., Tulej, M., Thomas, N., Wieser, M., Barabash, S., 2015, Realistic ice sputtering experiments for the surfaces of Galilean moons, EPSC Abstracts, 10, EPSC2015-771-1.

Galli, A., Vorburger, A., Pommerol, A., Wurz, P., Jost, B., Poch, O., Brouet, Y., Tulej, M., Thomas, N., 2016, Surface charging of thick porous water ice layers relevant for ion sputtering experiments, Planetary and Space Sciences, 126, 63-71.

Gibson, K.D., Killelea, D.R., Yuan, H.-Q., Becker, J.S., Sibener, S.J., 2011. Determination of the sticking coefficient and scattering dynamics of water on ice using molecular beam techniques. The Journal of Chemical Physics, 134, 034703.

Grundy, W.M., Buie, M.W., Stansberry, J.A., Spencer, J.R., 1999. NearInfrared Spectra of Icy Outer Solar System Surfaces: Remote Determination of $\mathrm{H}_{2} \mathrm{O}$ Ice Temperatures. Icarus 142, 536-549.

Hand, K.P., Carlson, R.W., 2011. $\mathrm{H}_{2} \mathrm{O}_{2}$ production by high-energy electrons on icy satellites as a function of surface temperature and electron flux. Icarus 215, 226-233.

Johnson, R.E., 1990. Energetic Charged-Particle Interactions with Atmospheres and Surfaces. Springer-Verlag, New York. 
Johnson, R.E., Quickenden, T.I., Cooper, P.D., McKinley, A.J., Freeman, C.G., 2003. The Production of Oxidants in Europa's Surface. Astrobiology $3,823-850$.

Johnson, R.E., Carlson, R.W., Cooper, J.F., Paranicas, C., Moore, M.H., Wong, M.C., 2004. Radiation effects on the surfaces of the Galilean satellites. In: Bagenal, F. (Ed.), Jupiter: Atmosphere, Satellites and Magnetosphere. University of Arizona Press, Tucson, USA.

Johnson, R.E., Burger, M.H., Cassidy, T.A., Leblanc, F., Marconi, M., Smyth, W.H., 2009. Composition and Detection of Europas SputterInduced Atmosphere. In: Pappalardo, R.T., McKinnon, W.B., Khurana, K.K. (Eds.), Europa. University of Arizona Press, Tucson.

Johnson, R.E. and Liu, M., 2010. Sputtering of Surfaces, Sputtering Data for $\mathrm{H}_{2} \mathrm{O}$ ice, http://people.virginia.edu/ rej/h2o.html

Johnson, R.E., Carlson, R.W., Cassidy, T.A., Famá, M., 2013. Sputtering of Ices. In: Gudipati, M.S. Castillo-Rogez, J. (Eds.), The Science of Solar System Ices. Springer, New York.

Küstner, M., Eckstein, W., Dose, V., Roth, J, 1998. The influence of sur${ }_{630}$ face roughness on the angular dependence of the sputter yield. Nuclear Instruments and Methods in Physical Research B 145, 320-331.

Marconi, M.L., 2007. A kinetic model of Ganymede's atmosphere. Icarus 190, 155.

Marti, A., Schletti, R., Wurz, P., Bochsler, P., 2001. Calibration facility for solar wind plasma instrumentation. Review of Scientific Instruments 72, 1354. doi:10.1063/1.1340020.

Mitchell, E.H., Raut, U., Teolis, B.D., Baragiola, R.A., 2016. Porosity effects on crystallization kinetics of amorphous solid water: Implications for cold icy objects in the outer solar system. Icarus, in press.

640

Muntean, E.A., Lacerda, P., Field, T.A., Fitzsimmons, A., Hunniford, C.A., McCullough, R.W., 2015. Sputtering of oxygen ice by low energy ions. Surface Science 641, 204-209. 
Muntean, E. A., Lacerda, P., Field, T. A., Fitzsimmons, A., Fraser, W. C., Hunniford, A. C., McCullough, R. W., 2016. A laboratory study of water ice erosion by low-energy ions. Monthly Notices of the Royal Astronomical Society, 462, 3361-3367.

Orlando, T.M., Sieger, M.T., 2003. The role of electron-stimulated production of $\mathrm{O}_{2}$ from water ice in the radiation processing of outer solar system surfaces. Surface Sciences 528, 1.

Paranicas, C., Mauk, B.H., Ratliff, J.M., Cohen, C., Johnson, R.E., 2002. The ion environment near Europa and its role in surface energetics. Geophysical Research Letters 29, 18.

Plainaki, C., Milillo, A., Mura, A., Orsini, S., Massetti, S., Cassidy, T., 2012. The role of sputtering and radiolysis in the generation of Europa exosphere. Icarus 218, 956.

Plainaki, C., Milillo, A., Massetti, S., Mura, A., Jia, X., Orsini, S., Mangano, V., De Angelis, E., Rispoli, R., 2015. The $\mathrm{H}_{2} \mathrm{O}$ and $\mathrm{O}_{2}$ exospheres of Ganymede: The result of a complex interaction between the jovian magnetospheric ions and the icy moon. Icarus 245, 306.

Reimann, C.T., Boring, J.W., Johnson, R.E., Garrett, J.W., Farmer, K.R., 1984. Ion-induced molecular ejection from $\mathrm{D}_{2} \mathrm{O}$ ice. Surface Science 147, $227-240$.

Sergis, N., Krimigis, S.M., Mitchell, D.G., Hamilton, D.C., Krupp, N., Mauk, B.H., Roelof, E.C., Dougherty, M.K., 2009. Energetic particle pressure in Saturn's magnetosphere measured with the Magnetospheric Imaging Instrument on Cassini. Journal of Geophysical Research, 114, A02214.

Shemansky, D.E., Yung, Y.L., Liu, X., Yoshii, J., Hansen, C.J., Hendrix, A.R., Esposito, L.W., 2014. A new understanding of the Europa atmosphere and limits on geophysical activity. The Astrophysical Journal, 797, 84 .

Shematovich, V.I., Johnson, R.E., Cooper, J.F., Wong, M.C., 2005. Surface bounded atmosphere of Europa. Icarus 173, 480-498. 
Shi, J., Famá, M., Teolis, B.D., Baragiola, R.A., 2010. Ion-induced electrostatic charging of ice. Nuclear Instruments and Methods in Physical Research B 268, 2888-2891.

Shi, J., Famá, M., Teolis, B.D., Baragiola, R.A., 2012. Ion-induced electrostatic charging of ice at 15-160 K. Physical Review B 85, 035424.

Teolis, B.D., Shi, J., Baragiola, R.A., 2009. Formation, trapping, and ejection of radiolytic $\mathrm{O}_{2}$ from ion-irradiated water ice studied by sputter depth profiling. The Journal of Chemical Physics 130, 134704.

Teolis, B.D., Plainaki, C., Cassidy, T.A., Raut, U., 2016. Quantification of $\mathrm{O}_{2}, \mathrm{H}_{2}$ and $\mathrm{H}_{2} \mathrm{O}_{2}$ radiolysis from ice for any projectile species, energy or temperature: Application to Icy Satellites. Submitted to Icarus

Vidal R.A., Teolis, B.D., Baragiola, R.A., 2005. Angular dependence of the 685 sputtering yield of water ice by $100 \mathrm{keV}$ proton bombardment. Surface Science 588, 1-5.

Wurz, P., 2000, Detection of energetic neutral particles, in "The Outer Heliosphere: Beyond the Planets", ed. by K. Scherer, H. Fichtner, E. Marsch (Copernicus Gesellschaft e.V., Katlenburg-Lindau, 2000), 251-288.

Ziegler, J.F., Biersack, J.P., Littmark, U., 1985. The stopping and range of ions in matter. Pergamon, New York.

Ziegler, J.F., Biersack, J.P., Ziegler, M. D., 2008. SRIM - The Stopping and Range of Ions in Matter, Vol. 5. SRIM Co., Chester, MD. 